TRANSACTIONS OF THE

AMERICAN MATHEMATICAL SOCIETY

Volume 362, Number 12, December 2010, Pages 6183-6203

S 0002-9947(2010)05056-1

Article electronically published on July 22, 2010

\title{
ANNIHILATORS OF GRADED COMPONENTS OF THE CANONICAL MODULE, AND THE CORE OF STANDARD GRADED ALGEBRAS
}

\author{
LOUIZA FOULI, CLAUDIA POLINI, AND BERND ULRICH
}

\begin{abstract}
We relate the annihilators of graded components of the canonical module of a graded Cohen-Macaulay ring to colon ideals of powers of the homogeneous maximal ideal. In particular, we connect them to the core of the maximal ideal. An application of our results characterizes Cayley-Bacharach sets of points in terms of the structure of the core of the maximal ideal of their homogeneous coordinate ring. In particular, we show that a scheme is Cayley-Bacharach if and only if the core is a power of the maximal ideal.
\end{abstract}

\section{INTRODUCTION}

This paper started as an attempt to understand the core of powers of the homogeneous maximal ideal of a standard graded algebra. The core of an arbitrary ideal $I$ in a Noetherian ring is defined as the intersection of all reductions of $I$, equivalently, of all ideals over which $I$ is integral. The definition simplifies when $I$ is a power $\mathfrak{m}^{n}$ of the homogeneous maximal ideal $\mathfrak{m}$ of a standard graded CohenMacaulay algebra $R$ of positive dimension over an infinite field $k$. In this case, $\operatorname{core}\left(\mathfrak{m}^{n}\right)$ is the intersection of all ideals generated by systems of parameters consisting of forms of degree $n$; in fact, it suffices to intersect finitely many parameter ideals generated by general forms of degree $n$ [3, 15]. One has explicit formulas in this case that express the core of $\mathfrak{m}^{n}$ as a colon ideal and that are valid in any characteristic. If char $k=0$ or if $R$ is geometrically reduced, then

$$
\operatorname{core}\left(\mathfrak{m}^{n}\right)=J^{j+(n-1) d+1}: \mathfrak{m}^{j},
$$

where $J$ is an ideal generated by a linear system of parameters of $R, j$ is any integer $\geq a+d$, and $a, d$ denote the $a$-invariant and the dimension of $R$, respectively (see Theorem [4.2 and, for prior results, [11, 7]). Thus the question arises of what more can be said about the colon ideals $J^{j+(n-1) d+1}: \mathfrak{m}^{j}$ and, most notably, when the obvious inclusion $\mathfrak{m}^{a+n d+1} \subset J^{j+(n-1) d+1}: \mathfrak{m}^{j}$ is an equality. We were also wondering in what sense the shape of the core of $\mathfrak{m}$ or its powers reflects the geometry of $\operatorname{Proj}(R)$ as a subscheme of projective space. As it turns out, the crucial device in approaching these questions is the graded canonical module $\omega$ of $R$ and the faithfulness of the submodules generated by its graded components.

Received by the editors August 14, 2007.

2010 Mathematics Subject Classification. Primary 13B21; Secondary 13A30, 13B22, 13C40.

The second and third author gratefully acknowledge partial support from the NSF. The second author was also supported in part by the NSA. The first and second author thank the Department of Mathematics of Purdue University for its hospitality.

(C)2010 American Mathematical Society Reverts to public domain 28 years from publication 
Thus the main goal of this paper is to study, quite broadly, the interplay between annihilators of graded components of $\omega$ on the one hand and colon ideals of powers of $\mathfrak{m}$ on the other hand.

More generally, let $R$ be a standard graded Cohen-Macaulay algebra over a field $k$, of dimension $d>0$, and write $\mathfrak{m}$ for its homogeneous maximal ideal and $\omega=\omega_{R}$ for its graded canonical module. By $a=a(R)$ we denote the $a$-invariant of $R$, which is the negative of the initial degree of $\omega$. Recall that if $J$ is any ideal generated by a linear system of parameters, then ' $J$ is a minimal reduction of $\mathfrak{m}$ with reduction number $r_{J}(\mathfrak{m})=a+d$ ', which simply means that $\mathfrak{m}^{i}=J^{i-j} \mathfrak{m}^{j}$ for every $i \geq j \geq a+d$, but for no smaller $j$. It is easy to see that $J^{i}: \mathfrak{m}^{j}=\mathfrak{m}^{i-j+a+d}$ for every $i$ and $j \geq a+d$, provided $R$ is Gorenstein or, more generally, level, which means that $\omega$ is generated by homogeneous elements of the same degree, $\omega=[\omega]_{-a} R$. Under these assumptions, the $R$-submodules $[\omega]_{t} R$ of $\omega$ generated by the homogeneous elements of a fixed degree $t$ are all faithful as long as $t \geq-a$; this is obvious since $\omega=[\omega]_{-a} R$ is faithful and there exists a form of positive degree regular on $\omega$. The same holds if $R$ is a domain because a suitable shift of $\omega$ embeds into $R$. Without the additional assumptions on the ring, neither the statement about the colon ideals nor the one about the graded components of the canonical module are true, but there is a close relationship between the two conditions. In fact in one of our main results we express, more generally, the annihilators of graded components of $\omega$ in terms of colon ideals of powers of $\mathfrak{m}$,

$$
\operatorname{ann}_{R}\left([\omega]_{\leq t} R\right)=\operatorname{ann}_{R}\left([\omega]_{t} R\right)=\bigoplus_{i}\left[J^{i+j-d+t+1}: \mathfrak{m}^{j}\right]_{i}
$$

for every $t$ and $j \geq a+d$ (see Theorem 2.7). Conversely, this allows one to write the colon ideals $J^{i}: \mathfrak{m}^{j}$ for any $i$ and $j \geq a+d$ in the form

$$
J^{i}: \mathfrak{m}^{j}=\mathfrak{m}^{i-j+a+d}+N,
$$

where $\mathfrak{m}^{i-j+a+d}$ is the 'expected' part and $N$ is an ideal of height zero that is generated in degrees $\leq i-j+a+d-1$ and can be described as

$$
N=\left(\bigoplus_{l \leq i-j+a+d-1}\left[\operatorname{ann}_{R}\left([\omega]_{i-j-l+d-1} R\right)\right]_{l}\right) R
$$

(see Corollaries 2.14 and 2.15). To prove these results it is useful to replace the colon ideals $J^{i}:_{R} \mathfrak{m}^{j}$ in $R$ by the corresponding colon ideals $J^{i} \omega: \omega \mathfrak{m}^{j}$ in $\omega$, which we then relate to truncations $[\omega]_{\geq i-j+d}$ of $\omega$ and to graded components of the canonical module $\Omega$ of the extended Rees ring of $\mathfrak{m}$. This is done in one of our main technical results. There we also use a bound on the regularity of $\Omega$ to show that $J^{i} \omega:_{\omega} \mathfrak{m}^{j}=J^{i-j}\left(J^{j} \omega: \omega \mathfrak{m}^{j}\right)$ for every integer $i$ with $i \geq j$ (see Theorem 2.3 and, for related results, 10, 14, 11, 4]).

Our results imply, for instance, that $[\omega]_{-a} R$ is faithful, equivalently, $[\omega]_{t} R$ is faithful for every $t \geq-a$, if and only if $J^{i}: \mathfrak{m}^{j}=\mathfrak{m}^{i-j+a+d}$ for every $i$ and $j \geq a+d$, if and only if $J^{i}: \mathfrak{m}^{a+d}=\mathfrak{m}^{i}$ for some $i \gg 0$ (see Corollary 2.10). The question arises how large the integer $i$ has to be chosen in the last statement. Conceivably $i=a+d+1$ always works at least when $k$ is perfect and $R$ is reduced, and we can show this if $d=1$ or if $R$ is an almost complete intersection of embedding codimension 2, for instance (see Corollaries 2.11 and 3.11). In general we can prove that $i=\alpha+d+1$ suffices, where $\alpha=\alpha(R)$ is the smallest possible $a$-invariant of a standard graded Gorenstein ring of dimension $d$ mapping onto $R$ so that the kernel 
vanishes locally at its minimal primes (see Corollary 3.8). We deduce this result from an estimate on the initial degree of certain colon ideals (see Proposition 3.5), which in turn follows from a general bound on the generic generator degree of the canonical module (see Proposition 3.1). The stronger result in the case of almost complete intersections of embedding codimension 2 is proved using an estimate for the generic generator degree of the first syzygy module of homogeneous ideals (see Proposition 3.2).

Returning to the core and the general assumptions used in this context, we conclude that $[\omega]_{-a} R$ is faithful if and only if core $\left(\mathfrak{m}^{n}\right)=\mathfrak{m}^{n d+a+1}$ for every $n \geq 1$ if and only if core $\left(\mathfrak{m}^{n}\right)$ is generated in one degree for some $n \gg 0$ (see Theorem4.4). Furthermore, core $\left(\mathfrak{m}^{n}\right)$ can be replaced by core $(\mathfrak{m})$ in the last statement, provided $d=1$ or $R$ is a reduced almost complete intersection of embedding codimension 2 , for instance (see Corollary 4.5). This leads, rather directly, to a geometric interpretation of the core when $R$ is the homogeneous coordinate ring of a finite set $X$ of reduced points in projective space. Most notably, $\operatorname{core}(\mathfrak{m})=\mathfrak{m}^{a+2}$ if and only if $X$ has the Cayley-Bacharach property (see Corollary 5.5). Recall that $X$ is Cayley-Bacharach if the Hilbert function of $X \backslash\{P\}$ does not depend on the point $P \in X$. Since this property is equivalent to the faithfulness of $[\omega]_{-a} R$ (see $[8]$ ), the above characterization in terms of the core then follows as an immediate consequence of Corollary 4.5. We also show that if a large enough subset of $X$ lies on a hypersurface of low degree, then the initial degree of core $(\mathfrak{m})$ is forced to be unexpectedly small (see Corollary 5.4 and Proposition 6.1), underlining once more the fact that the shape of the core reflects uniformity properties of the set of points, or the lack thereof.

\section{What annihilates the COMPONENTS of ThE CANONICAL MOdUle?}

We begin by fixing notation and recalling some general facts. Let $k$ be a field and $R$ a standard graded $k$-algebra of dimension $d$ with homogeneous maximal ideal $\mathfrak{m}$ and graded canonical module $\omega$. Recall that

$$
a(R)=-\min \left\{i \mid[\omega]_{i} \neq 0\right\}
$$

is the a-invariant of $R$. We also consider the integers

$$
\begin{aligned}
& b(R)=-\min \left\{i \mid[\omega]_{i} R \text { is } R \text {-faithful }\right\} \text { and } \\
& c(R)=-\max \left\{i \mid\left[k \otimes_{R} \omega\right]_{i} \neq 0\right\} .
\end{aligned}
$$

By local duality, the $a$-invariant is the top degree of the local cohomology module $H_{\mathfrak{m}}^{d}(R)$. Also notice that $c(R)$ is the negative of the largest generator degree of $\omega$. Since $\omega$ is $R$-faithful we have $a(R) \geq b(R) \geq c(R)$.

Now assume that $R$ is Cohen-Macaulay. Let $S=k\left[x_{1}, \ldots, x_{n}\right]$ be a polynomial ring mapping homogeneously onto $R$ and write $g=\operatorname{codim}_{S} R=\operatorname{projdim}_{S} R$. The integers $a(R)$ and $c(R)$ can be expressed in terms of the minimal homogeneous free $S$-resolution of $R$ by means of the formulas

$$
\begin{aligned}
& a(R)=\max \left\{i \mid\left[\operatorname{Tor}_{g}^{S}(k, R)\right]_{i} \neq 0\right\}-n, \\
& c(R)=\min \left\{i \mid\left[\operatorname{Tor}_{g}^{S}(k, R)\right]_{i} \neq 0\right\}-n .
\end{aligned}
$$

Notice that $c(R) \geq-d$; in particular, $\omega$ is generated in degrees at most $d$ and $a(R)+d \geq 0$. 
We also consider the extended Rees algebra $R\left[\mathfrak{m} t, t^{-1}\right]$, which is a bigraded subring of $R\left[t, t^{-1}\right]$. There are natural maps of bigraded modules

$\omega_{R\left[\mathfrak{m} t, t^{-1}\right]} \hookrightarrow\left(\omega_{R\left[\mathfrak{m} t, t^{-1}\right]}\right)_{t^{-1}} \cong \omega_{R\left[\mathfrak{m} t, t^{-1}\right]_{t^{-1}}}=\omega_{R\left[t, t^{-1}\right]} \cong \omega \otimes_{R} R\left[t, t^{-1}\right]=\bigoplus \omega t^{i}$.

Identifying $\omega_{R\left[\mathfrak{m} t, t^{-1}\right]}$ with its image in $\bigoplus \omega t^{i}$ we obtain a bigraded canonical module

$$
\Omega=\bigoplus \Omega_{i} t^{i} \subset \bigoplus \omega t^{i}
$$

so that $\Omega_{i}=\omega$ for $i \ll 0$. One has homogeneous isomorphisms $R\left[\mathfrak{m} t, t^{-1}\right] /\left(t^{-1}\right) \cong$ $\operatorname{gr}_{\mathfrak{m}}(R) \cong R$, thinking of the last $R$ as being diagonally bigraded. In particular $R\left[\mathfrak{m} t, t^{-1}\right]$ is Cohen-Macaulay. Hence the above isomorphisms induce identifications

$$
\Omega / t^{-1} \Omega \cong \omega(1) \text { and } \Omega_{i} / \Omega_{i+1} \cong[\omega]_{i+1} .
$$

We will use the convention that the power of any element or ideal with nonpositive exponent is one or the unit ideal, respectively.

Assumptions 2.1. We assume $k$ is a field and $R$ is a standard graded CohenMacaulay $k$-algebra of dimension $d \geq 1$ with homogeneous maximal ideal $\mathfrak{m}$ and graded canonical module $\omega$. We write $a=a(R), b=b(R), c=c(R)$, and let $\Omega=\omega_{R\left[\mathfrak{m} t, t^{-1}\right]}$ be as above, with $\Omega_{i}=\omega$ for $i \ll 0$. Let $y_{1}, \ldots, y_{d}$ be a system of parameters in $R$ consisting of linear forms. We write $J$ for the ideal generated by $y_{1}, \ldots, y_{d}$ and $J^{[i]}$ for the ideal generated by the powers $y_{1}^{i}, \ldots, y_{d}^{i}$, where $i$ is any integer.

Elements $y_{1}, \ldots, y_{d}$ as in Assumptions 2.1 always exist if $k$ is infinite. The ideal $J$ they generate is a minimal reduction of $\mathfrak{m}$ with reduction number $r_{J}(\mathfrak{m})=a+d$, as can be seen by reducing modulo $J$. Therefore

$$
J^{i}: \mathfrak{m}^{j}=J^{[i]}: \mathfrak{m}^{j+(i-1)(d-1)}
$$

for every $i$ and $j \geq a+d$; in fact, one can show as in [15, proof of 2.2] that if $I$ is any $\mathfrak{m}$-primary ideal and $\beta_{1}, \ldots, \beta_{d}$ are elements of $I$ with $I^{j+1}=\left(\beta_{1}, \ldots, \beta_{d}\right) I^{j}$ for some integer $j$, then

$$
\left(\beta_{1}, \ldots, \beta_{d}\right)^{i}: I^{j}=\left(\beta_{1}^{i}, \ldots, \beta_{d}^{i}\right): I^{j+(i-1)(d-1)}
$$

for every $i$.

In this section we prove our results about the relationship between annihilators of graded components of $\omega$ on the one hand and the colon ideals $J^{i}: \mathfrak{m}^{j}$ on the other hand. Here it suffices to consider $j=a+d$, as the next remark shows.

Remark 2.2. With assumptions as in 2.1, one has

$$
J^{i}: \mathfrak{m}^{j}=J^{i-j+a+d}: \mathfrak{m}^{a+d}
$$

for every $i$ and $j \geq a+d$.

Proof. Since $j \geq a+d=r_{J}(\mathfrak{m})$, we obtain

$$
J^{i}: \mathfrak{m}^{j}=J^{i}: J^{j-a-d} \mathfrak{m}^{a+d}=\left(J^{i}: J^{j-a-d}\right): \mathfrak{m}^{a+d}=J^{i-j+a+d}: \mathfrak{m}^{a+d} .
$$

The last equality holds because the associated graded ring of $J$ has positive depth. 
We first study the colons $J^{i} \omega: \omega \mathfrak{m}^{j}$ in $\omega$, which exhibit a more regular behavior than the corresponding ideals $J^{i}:_{R} \mathfrak{m}^{j}$. We will write $[\omega]_{\geq l}=\bigoplus_{i \geq l} \omega_{i}$ and $[\omega]_{\leq l}=$ $\bigoplus_{i \leq l} \omega_{i}$

Theorem 2.3. In addition to the assumptions of 2.1, let $i$ and $j \geq a+d$ be integers, and set $s=j+(i-1)(d-1)$. One has

(a) $J^{i} \omega: \omega \mathfrak{m}^{j}=J^{[i]} \omega: \omega \mathfrak{m}^{s}=[\omega]_{\geq i-j+d}=\Omega_{i-j+d-1}$;

(b) $J^{i} \omega: \omega \mathfrak{m}^{j}=J^{i-j}\left(J^{j} \omega: \omega \mathfrak{m}^{j}\right)$ whenever $i \geq j$.

Proof. To prove part (a) we first notice that

$$
\Omega_{i-j+d-1}=J^{i} \omega: \omega \mathfrak{m}^{j}
$$

according to 4]. Next,

$$
\begin{array}{rlrl}
J^{i} \omega: \omega \mathfrak{m}^{j} & =\left(J^{[i]}:_{R} J^{(i-1)(d-1)}\right) \omega: \mathfrak{m}^{j} & & \text { by (2.2) } \\
& \subset\left(J^{[i]} \omega:_{\omega} J^{(i-1)(d-1)}\right):_{\omega} \mathfrak{m}^{j} & \\
& =J^{[i]} \omega: \omega\left(J^{(i-1)(d-1)} \mathfrak{m}^{j}\right) & \\
& =J^{[i]} \omega: \omega \mathfrak{m}^{s} & & \text { because } j \geq a+d=r_{J}(\mathfrak{m}) .
\end{array}
$$

To show that $J^{[i]} \omega: \omega \mathfrak{m}^{s}=[\omega]_{\geq i-j+d}$ we may reduce modulo $J^{[i]} \omega$; indeed, $J^{[i]} \omega \subset[\omega]_{\geq i-j+d}$ because $\omega$ is concentrated in degrees $\geq-a$ and $i-a \geq i-j+d$. However, $\left(J^{[i]} \omega: \omega \mathfrak{m}^{s}\right) / J^{[i]} \omega \cong \overline{0}: \bar{\omega} \overline{\mathfrak{m}}^{s}$, where $\overline{\mathfrak{m}}$ is the homogeneous maximal ideal of $\bar{R}=R / J^{[i]}$ and $\bar{\omega}=\omega / J^{[i]} \omega \cong \omega_{\bar{R}}(-i d)$. If $i \leq 0$ then $\bar{\omega}=0$, and we are done. Otherwise $\omega_{\bar{R}}$ is an Artinian module with socle concentrated in degree 0 , and then $\bar{\omega}$ is Artinian with socle concentrated in degree $i d$. Furthermore $J \geq 0$. Thus $\overline{0}:_{\bar{\omega}} \overline{\mathfrak{m}}^{s}=[\bar{\omega}]_{\geq i d+1-s}=[\bar{\omega}]_{\geq i-j+d}$. It follows that $J^{[i]} \omega:_{\omega} \mathfrak{m}^{s}=[\omega]_{\geq i-j+d}$.

So far we have shown that

$$
\Omega_{i-j+d-1}=J^{i} \omega: \omega \mathfrak{m}^{j} \subset J^{[i]} \omega: \omega \mathfrak{m}^{s}=[\omega]_{\geq i-j+d} .
$$

Now $\Omega=\bigoplus \Omega_{l} t^{l} \subset \bigoplus[\omega]_{\geq l+1} t^{l}=\Omega^{\prime}$ are bigraded $R\left[\mathfrak{m} t, t^{-1}\right]$-modules that are finitely generated because $[\omega]_{\geq l+1}=\mathfrak{m}[\omega]_{\geq l}$ for $l \gg 0$. By (2.1) this inclusion induces an isomorphism $\Omega / t^{-1} \Omega \cong \Omega^{\prime} / t^{-1} \Omega^{\prime}$, which shows that $\Omega^{\prime}=\Omega+t^{-1} \Omega^{\prime}$. Therefore $\Omega^{\prime}=\Omega$ by the graded Nakayama lemma.

We now prove part (b). In light of (a) we need to show that for every $l \geq d$ one has $[\omega]_{\geq l+1}=J[\omega]_{\geq l}$, or equivalently, $[\omega]_{\geq l+1} \subset J \omega$. However, $\omega / J \omega \cong \omega_{R / J}(-d)$ is concentrated in degrees $\leq d$, which gives $[\omega]_{\geq l+1} \subset J \omega$.

The next result addresses the comparison between the colons $J^{i} \omega: \omega \mathfrak{m}^{j}$ and $J^{i}:_{R} \mathfrak{m}^{j}$.

Corollary 2.4. In addition to the assumptions of [2.1, let $i$ and $j \geq a+d$ be integers. If

$$
J^{i} \omega:_{\omega} \mathfrak{m}^{j}=\left(J^{i}:_{R} \mathfrak{m}^{j}\right) \omega \quad \text { for some } i \geq j,
$$

then for every $l \geq i$,

(a) $J^{l} \omega: \omega \mathfrak{m}^{j}=\left(J^{l}:_{R} \mathfrak{m}^{j}\right) \omega ;$

(b) $J^{l}:_{R} \mathfrak{m}^{j}$ is integral over $J^{l-i}\left(J^{i}:_{R} \mathfrak{m}^{j}\right)$; in particular, if $R$ is reduced, then the initial degree of the former ideal satisfies

$$
\operatorname{indeg}\left(J^{l}:_{R} \mathfrak{m}^{j}\right)=\operatorname{indeg}\left(J^{i}:_{R} \mathfrak{m}^{j}\right)+l-i .
$$


Proof. One has

$$
\begin{array}{rlrl}
\left(J^{l}:_{R} \mathfrak{m}^{j}\right) \omega & \subset J^{l} \omega: \omega \mathfrak{m}^{j} & \\
& =J^{l-i}\left(J^{i} \omega:_{\omega} \mathfrak{m}^{j}\right) & & \text { by Theorem 2.3(b) } \\
& =J^{l-i}\left(J^{i}:_{R} \mathfrak{m}^{j}\right) \omega & & \text { by our assumption } \\
& \subset\left(J^{l}:_{R} \mathfrak{m}^{j}\right) \omega . & &
\end{array}
$$

This immediately gives $J^{l} \omega: \omega \mathfrak{m}^{j}=\left(J^{l}:_{R} \mathfrak{m}^{j}\right) \omega$, proving assertion (a). Furthermore, $\left(J^{l}:_{R} \mathfrak{m}^{j}\right) \omega=J^{l-i}\left(J^{i}:_{R} \mathfrak{m}^{j}\right) \omega$, which implies (b) since $\omega$ is a faithful $R$-module.

The next proposition gives a characterization for when the equality assumed in the previous corollary obtains.

Proposition 2.5. In addition to the assumptions of 2.1, let $i$ and $j \geq a+d$ be integers. Set $s=j+(i-1)(d-1)$ and let ${ }^{-}$denote images in $\bar{R}=R / J^{[i]}$. One has

$$
J^{i} \omega: \omega \mathfrak{m}^{j}=\left(J^{i}:_{R} \mathfrak{m}^{j}\right) \omega
$$

if and only if

$$
\overline{\mathfrak{m}}^{s}=\overline{0}: \bar{R}\left(\overline{0}: \overline{\bar{m}^{s}} \overline{\mathfrak{m}}^{s} .\right.
$$

Proof. First notice that $\bar{R}$ is an Artinian ring and that $\bar{\omega}=\omega / J^{[i]} \omega \cong \omega_{\bar{R}}(-i d)$. From Theorem 2.3(a) one has $J^{i} \omega: \omega \mathfrak{m}^{j}=J^{[i]} \omega: \omega \mathfrak{m}^{s}$. Hence it follows that $\left(J^{i} \omega: \omega \mathfrak{m}^{j}\right) / J^{[i]} \omega \cong \overline{0}: \bar{\omega} \overline{\mathfrak{m}}^{s}$. Now the latter module is naturally isomorphic to $\left(\overline{0}: \omega_{\bar{R}} \overline{\mathfrak{m}}^{s}\right)(-i d) \cong \omega_{\bar{R} / \overline{\mathfrak{m}}^{s}}(-i d)$. Thus we have shown that

$$
\left(J^{i} \omega: \omega \mathfrak{m}^{j}\right) / J^{[i]} \omega \cong \omega_{\bar{R} / \overline{\mathfrak{m}}^{s}}(-i d) .
$$

On the other hand, from (2.2) one knows that $J^{i}: \mathfrak{m}^{j}=J^{[i]}: \mathfrak{m}^{s}$. Therefore $\left(J^{i}:_{R} \mathfrak{m}^{j}\right) \omega / J^{[i]} \omega=\left(\overline{0}: \bar{R} \overline{\mathfrak{m}}^{s}\right) \bar{\omega}$, which is isomorphic to $\left(\overline{0}: \bar{R}_{\bar{R}} \overline{\mathfrak{m}}^{s}\right) \omega_{\bar{R}}(-i d)$. The last module can be identified with $\omega_{\bar{R} / \overline{0}:\left(\overline{0}: \overline{\mathbf{m}}^{s}\right)}(-i d)$, as shown in [16, 2.3(b)]. Hence we have proved that

$$
\left(J^{i}:_{R} \mathfrak{m}^{j}\right) \omega / J^{[i]} \omega \cong \omega_{\bar{R} / \overline{0}:\left(\overline{0}: \overline{\mathfrak{m}}^{s}\right)}(-i d) .
$$

We conclude that the obvious containment $\left(J^{i}:_{R} \mathfrak{m}^{j}\right) \omega \subset J^{i} \omega: \omega \mathfrak{m}^{j}$ is an equality if and only if the inclusion $\overline{\mathfrak{m}}^{s} \subset \overline{0}:\left(\overline{0}: \overline{\mathfrak{m}}^{s}\right)$ is.

If the equalities of Proposition 2.5 hold for all $i$, then the canonical module of the extended Rees ring of $\mathfrak{m}$ has an easy description, namely

$$
\Omega=\left(R\left[J t, t^{-1}\right]:_{R\left[t, t^{-1}\right]} \mathfrak{m}^{j}\right) t^{d-1-j} \omega
$$

(see Theorem [2.3(a) or [4]). These equalities obtain, quite generally, when $i \geq j$ and $d=1$ :

Proposition 2.6. In addition to the assumptions of 2.1, suppose that $d=1$ and $R$ is reduced. For every $i \geq j$ and $j \geq a+1$ one has

$$
\left(J^{i}:_{R} \mathfrak{m}^{j}\right) \omega=J^{i} \omega: \omega \mathfrak{m}^{j}=[\omega]_{\geq i-j+1} .
$$

Proof. The second equality follows from Theorem $2.3(\mathrm{a})$. We prove the first equality. It suffices to consider the case $i=j=a+1$ according to Remark 2.2 and 
Corollary 2.4 (a), or simply because $J$ is generated by a single regular element. In light of Proposition 2.5 we need to show that

$$
\mathfrak{m}^{a+1}=y^{a+1} R:_{R}\left(y^{a+1} R:_{R} \mathfrak{m}^{a+1}\right)
$$

with $y=y_{1}$.

In the total ring of quotients $K$ of $R$ we consider the integral closure $S$ of $R$. Our assumptions, most notably the reducedness of $R$, imply that $R \subset S$ is a homogeneous inclusion of nonnegatively graded Noetherian rings and moreover $R:_{K}\left(R:_{K} S\right)=S$. Thus we obtain

$$
\begin{aligned}
\mathfrak{m}^{a+1} & \subset y^{a+1} R:_{R}\left(y^{a+1} R:_{R} \mathfrak{m}^{a+1}\right) \\
& \subset y^{a+1} R:_{R}\left(y^{a+1} R:_{R} \mathfrak{m}^{a+1} S\right) \\
& =y^{a+1} R:_{R}\left(y^{a+1} R:_{R} y^{a+1} S\right) \\
& =y^{a+1} R:_{R}\left(R:_{R} S\right) \\
& =\left[y^{a+1} R:_{K}\left(R:_{R} S\right)\right] \cap R \\
& =\left[y^{a+1}\left(R:_{K}\left(R:_{K} S\right)\right)\right] \cap R \\
& =y^{a+1} S \cap R \\
& \subset \mathfrak{m}^{a+1}
\end{aligned}
$$

since $y R$ is a reduction of $\mathfrak{m}$ since $y$ is $R$-regular

since $R:_{K}\left(R:_{K} S\right)=S$

since $S$ is nonnegatively graded.

Next we are going to use the above results to say something about the annihilators of the graded components of $\omega$.

Theorem 2.7. With assumptions as in 2.1, one has

$$
\operatorname{ann}_{R}\left([\omega]_{\leq t} R\right)=\operatorname{ann}_{R}\left([\omega]_{t} R\right)=\bigoplus_{i}\left[J^{i+j-d+t+1}: \mathfrak{m}^{j}\right]_{i}
$$

for every $t$ and $j \geq a+d$.

Proof. The first equality is obvious because $R$ contains a linear form, namely $y_{1}$, that is regular on $\omega$.

Theorem 2.3(a) shows that

$$
\left(J^{i+j-d+t+1}: \mathfrak{m}^{j}\right) \omega \subset[\omega]_{\geq i+t+1},
$$

which gives $\left[J^{i+j-d+t+1}: \mathfrak{m}^{j}\right]_{i}[\omega]_{\leq t}=0$. This proves the inclusion

$$
\bigoplus_{i}\left[J^{i+j-d+t+1}: \mathfrak{m}^{j}\right]_{i} \subset \operatorname{ann}_{R}\left([\omega]_{\leq t} R\right) .
$$

To show the containment

$$
\operatorname{ann}_{R}\left([\omega]_{\leq t} R\right) \subset \bigoplus_{i}\left[J^{i+j-d+t+1}: \mathfrak{m}^{j}\right]_{i}
$$

we choose an element $f \in\left[\operatorname{ann}_{R}\left([\omega]_{\leq t} R\right)\right]_{i}$. We need to prove that

$$
f \in J^{i+j-d+t+1}: \mathfrak{m}^{j}=J^{[i+j-d+t+1]}: \mathfrak{m}^{j+(i+j-d+t)(d-1)},
$$

where the last equality holds by (2.2). Write $s=j+(i+j-d+t)(d-1)$ and let - denote images in the Artinian ring $\bar{R}=R / J^{[i+j-d+t+1]}$. The asserted inclusion $f \mathfrak{m}^{s} \subset J^{[i+j-d+t+1]}$ is equivalent to $\bar{f} \overline{\mathfrak{m}}^{s}=\overline{0}$, which in turn means that $\bar{f} \overline{\mathfrak{m}}^{s} \omega_{\bar{R}}=\overline{0}$ as $\omega_{\bar{R}}$ is faithful over $\bar{R}$. Since $\omega_{\bar{R}}=\bar{R} \otimes_{R} \omega((i+j-d+t+1) d)$ and $f[\omega]_{\leq t}=0$ 
it follows that $\bar{f}\left[\omega_{\bar{R}}\right]_{\leq t-(i+j-d+t+1) d}=\overline{0}$. Therefore $\bar{f}\left[\omega_{\bar{R}}\right]_{\leq-s-i}=\overline{0}$, and as $\bar{f}$ has degree $i$ we conclude that

$$
\bar{f} \overline{\mathfrak{m}}^{s} \omega_{\bar{R}} \subset\left[\omega_{\bar{R}}\right]_{\geq 1}=\overline{0} .
$$

Again, one has a better result in dimension one.

Corollary 2.8. In addition to the assumptions of 2.1, suppose that $d=1$ and write $y=y_{1}$. One has

$$
\operatorname{ann}_{R}\left([\omega]_{\leq t} R\right)=\operatorname{ann}_{R}\left([\omega]_{t} R\right)=\bigoplus_{i \leq-t-1}\left[J^{i+j+t}: \mathfrak{m}^{j}\right]_{i} \oplus\left[J^{j}: \mathfrak{m}^{j}\right]_{-t} k[y]
$$

for every $t$ and $j \geq a+1$.

Proof. We apply Theorem 2.7 using the fact that $J^{i+j+t}: \mathfrak{m}^{j}=y^{i+t}\left(J^{j}: \mathfrak{m}^{j}\right)$ for every $i \geq-t$.

The above corollary shows in particular that if $d=1$, then the graded $R$-module $\operatorname{ann}_{R}\left([\omega]_{\leq t} R\right)=\operatorname{ann}_{R}\left([\omega]_{t} R\right)$ has Castelnuovo-Mumford regularity at most $-t$; see also Proposition [3.9. For the definition and basic properties of the CastelnuovoMumford regularity we refer to [1, p.168].

We are now ready to answer one of the main questions raised in the introduction. With the next three corollaries we characterize the faithfulness of submodules generated by graded components of $\omega$, in terms of certain colon ideals of powers of $\mathfrak{m}$ having an 'expected form'. In light of Remark 2.2 we may restrict ourselves to the case $j=a+d$.

Corollary 2.9. With assumptions as in 2.1, the following are equivalent for an integer $t$ :

(a) $[\omega]_{t} R$ is faithful, i.e., $t \geq-b$;

(b) $J^{i+a+t}: \mathfrak{m}^{a+d} \subset \mathfrak{m}^{i}$ for every $i$;

(c) $J^{i+a+t}: \mathfrak{m}^{a+d} \subset \mathfrak{m}^{i}$ for some $i \gg 0$.

If $R$ is reduced and $J^{i+a+t} \omega::_{\omega} \mathfrak{m}^{a+d}=\left(J^{i+a+t}:_{R} \mathfrak{m}^{a+d}\right) \omega$ for some $i \geq d-t$, then the above conditions are equivalent to:

(d) $J^{i+a+t}: \mathfrak{m}^{a+d} \subset \mathfrak{m}^{i}$.

Proof. Recall that $R$ contains a linear form that is a regular element. Thus, if a homogeneous ideal vanishes in a certain degree it also vanishes in every smaller degree. Now Theorem 2.7 gives the equivalence of (a) and (b). The same theorem shows that if (c) holds, then $\left[\operatorname{ann}_{R}\left([\omega]_{t} R\right)\right]_{i-1}=0$ for some $i-1 \gg 0$, proving (a). Finally, (d) implies (c) according to Corollary 2.4(b).

Corollary 2.10. With assumptions as in 2.1, the following are equivalent:

(a) $[\omega]_{-a} R$ is faithful;

(b) $J^{i}: \mathfrak{m}^{a+d}=\mathfrak{m}^{i}$ for every $i$;

(c) $J^{i}: \mathfrak{m}^{a+d}=\mathfrak{m}^{i}$ for some $i \gg 0$.

If $R$ is reduced and $J^{i} \omega:_{\omega} \mathfrak{m}^{a+d}=\left(J^{i}:_{R} \mathfrak{m}^{a+d}\right) \omega$ for some $i \geq a+d$, then the above conditions are equivalent to :

(d) $J^{i}: \mathfrak{m}^{a+d}=\mathfrak{m}^{i}$. 
Proof. Notice that $\mathfrak{m}^{i} \subset J^{i}: \mathfrak{m}^{a+d}$ for every $i$ because $a+d=r_{J}(\mathfrak{m})$. Now the assertions follow from Corollary 2.9.

Corollary 2.11. In addition to the assumptions of 2.1 , suppose that $d=1$. The following are equivalent :

(a) $[\omega]_{-a} R$ is faithful;

(b) $J^{i}: \mathfrak{m}^{a+1}=\mathfrak{m}^{i}$ for every $i$;

(c) $J^{i}: \mathfrak{m}^{a+1}=\mathfrak{m}^{i}$ for some $i \geq a+1$.

Proof. In light of Corollary 2.10 it suffices to prove that if $J^{i}: \mathfrak{m}^{a+1}=\mathfrak{m}^{i}$ for some $i \geq a+1$, then $J^{l}: \mathfrak{m}^{a+1}=\mathfrak{m}^{l}$ for every $l \geq i$. Indeed, since $J$ is generated by a single regular element and $i \geq a+1=r_{J}(\mathfrak{m})$, it follows that

$$
J^{l}: \mathfrak{m}^{a+1}=J^{l-i}\left(J^{i}: \mathfrak{m}^{a+1}\right)=J^{l-i} \mathfrak{m}^{i}=\mathfrak{m}^{l} .
$$

The faithfulness of $[\omega]_{-a} R$, together with the additional condition $J^{i} \omega: \mathfrak{m}^{a+d}=$ $\left(J^{i}:_{R} \mathfrak{m}^{a+d}\right) \omega$ in Corollary 2.10 , means that $\omega$ and $[\omega]_{-a} R$ coincide from degree $i-a$ on:

Remark 2.12. In addition to the assumptions of 2.1, let $i \geq a+d$ be an integer. Then the equality $[\omega]_{\geq i-a}=\mathfrak{m}^{i}[\omega]_{-a}$ holds if and only if $J^{i} \omega:_{\omega} \mathfrak{m}^{a+d}=\left(J^{i}:_{R} \mathfrak{m}^{a+d}\right) \omega$ and $[\omega]_{-a} R$ is faithful. The forward direction follows because $[\omega]_{\geq i-a}=J^{i} \omega: \omega$ $\mathfrak{m}^{a+d}$ according to Theorem $2.3(\mathrm{a}), \mathfrak{m}^{i} \subset J^{i}: \mathfrak{m}^{a+d}$, and $[\omega]_{\geq i-a}$ is faithful. For the converse notice that Corollary 2.10 gives $J^{i}: \mathfrak{m}^{a+d}=\mathfrak{m}^{i}$; hence $[\omega]_{\geq i-a}=J^{i} \omega: \omega$ $\mathfrak{m}^{a+d}=\mathfrak{m}^{i} \omega$. Therefore $[\omega]_{\geq i-a}=\mathfrak{m}^{i}[\omega]_{-a}+[\omega]_{\geq i+1-a}$. However, $\omega$ is generated in degrees at most $d \leq i-a$; hence $[\omega]_{\geq i-a}$ is generated in degree $i-a$. It follows that $[\omega]_{\geq i-a}=\mathfrak{m}^{i}[\omega]_{-a}$.

Unfortunately, the faithfulness of $[\omega]_{-a} R$ alone is not sufficient to guarantee the equality $J^{i} \omega:_{\omega} \mathfrak{m}^{a+d}=\left(J^{i}:_{R} \mathfrak{m}^{a+d}\right) \omega$, as the next example shows.

Example 2.13. In addition to the assumptions of 2.1, suppose that $R$ is a domain of type 2 which is not level and which is not Gorenstein locally on the punctured spectrum. Clearly $[\omega]_{-a} R$ is faithful since $R$ is a domain. However, $J^{i} \omega:_{\omega} \mathfrak{m}^{a+d} \neq$ $\left(J^{i}:_{R} \mathfrak{m}^{a+d}\right) \omega$ for every $i \geq a+d$, because otherwise Remark 2.12 implies that $\omega$ and the cyclic module $[\omega]_{-a} R$ would coincide locally on the punctured spectrum.

Conversely, Theorem 2.7 can be used to obtain information about the colon ideals $J^{i}: \mathfrak{m}^{j}$. This is done in the remaining corollaries of this section.

Corollary 2.14. With assumptions as in 2.1, one has

$$
J^{i}: \mathfrak{m}^{j}=\mathfrak{m}^{i-j+a+d} \oplus \bigoplus_{l=i-j+b+d}^{i-j+a+d-1}\left[\operatorname{ann}_{R}\left([\omega]_{i-j-l+d-1} R\right)\right]_{l}
$$

for every $i$ and $j \geq a+d$.

Proof. Theorem 2.7 shows that

$$
\left[\operatorname{ann}_{R}\left([\omega]_{i-j-l+d-1} R\right)\right]_{l}=\left[J^{i}: \mathfrak{m}^{j}\right]_{l}
$$

for every $l$. On the other hand, $\left[\operatorname{ann}_{R}\left([\omega]_{i-j-l+d-1} R\right)\right]_{l}=0$ for $l \leq i-j+b+d-1$ by the definition of $b$. Finally, $\mathfrak{m}^{i-j+a+d} \subset J^{i}: \mathfrak{m}^{j}$. 
Corollary 2.15. With assumptions as in 2.1, there exists an ideal $N$ of height zero such that

$$
J^{i}: \mathfrak{m}^{j}=\mathfrak{m}^{i-j+a+d}+N
$$

for every $i$ and $j \geq a+d$.

Proof. Set $N=\left(\bigoplus_{l=i-j+b+d}^{i-j+a+d-1}\left[\operatorname{ann}_{R}\left([\omega]_{i-j-l+d-1} R\right)\right]_{l}\right) R$. According to Corollary 2.14, $J^{i}: \mathfrak{m}^{j}=\mathfrak{m}^{i-j+a+d}+N$. On the other hand, the first equality of Theorem 2.7 shows that $N \subset \operatorname{ann}_{R}\left([\omega]_{-a} R\right)$. The latter ideal has height zero since $[\omega]_{-a} R$ is a nonzero submodule of the maximal Cohen-Macaulay module $\omega$.

Corollary 2.16. In addition to the assumptions of [2.1, let $i$ and $j \geq a+d$ be integers. If $J^{i}: \mathfrak{m}^{j}$ is generated in one degree, then $J^{i}: \mathfrak{m}^{j}=\mathfrak{m}^{i-j+a+d}$.

Proof. The claim follows from Corollary 2.15 since $J^{i}: \mathfrak{m}^{j}$ has positive height.

Corollary 2.17. With assumptions as in 2.1 , one has

$$
\mathfrak{m}^{i-j+a+d} \subset J^{i}: \mathfrak{m}^{j} \subset \mathfrak{m}^{i-j+b+d}
$$

for every $i$ and $j \geq a+d$.

Proof. The containments are direct consequences of Corollary 2.14.

Corollary 2.18. In addition to the assumptions of [2.1, assume that $R$ is a domain or $R$ is level. One has

$$
J^{i}: \mathfrak{m}^{j}=\mathfrak{m}^{i-j+a+d}
$$

for every $i$ and $j \geq a+d$.

Proof. The result follows from Corollaries 2.15 and 2.17.

In the one-dimensional case the colon ideals $J^{i}: \mathfrak{m}^{j}$ can be expressed in terms of conductor ideals:

Remark 2.19. In addition to the assumptions of 2.1, suppose that $d=1$, write $y=y_{1}$, let $K$ denote the total ring of quotients of $R$, and let $R[\mathfrak{m} / y] \subset B$ be a homogeneous inclusion, where $B$ is a nonnegatively graded finite $R[\mathfrak{m} / y]$-module contained in $K$. One has $B=R[\mathfrak{m} / y]$ and

$$
J^{i}:_{R} \mathfrak{m}^{j}=J^{i-j}\left(R:_{K} B\right)=\mathfrak{m}^{i-j}\left(R:_{K} B\right)
$$

for every $i \geq j$ and $j \geq a+1$.

To prove the first claim notice that $B$ is a finite $R$-module and hence $B_{\geq l}=R_{\geq l}$ for $l \gg 0$. Therefore $B \geq l=\mathfrak{m}^{l}$, which gives $B \subset \mathfrak{m}^{l} / y^{l}$. As $j \geq a+1=r_{J}(\mathfrak{m})$ one has $\mathfrak{m}^{l} / y^{l}=\mathfrak{m}^{j} / y^{j}$. It follows that $B=\mathfrak{m}^{j} / y^{j}=R[\mathfrak{m} / y]$. The remaining assertions obtain because $J^{j}:_{R} \mathfrak{m}^{j}=y^{j} R:_{K} \mathfrak{m}^{j}=R:_{K} B$ and this is a $B$-module (see also [14, proof of 3.2]).

\section{INITIAL DEGREES OF ANNIHILATORS}

The previous section leaves open the question of how large the integer $i$ has to be chosen in Corollaries 2.9(c) and 2.10(c). Addressing this issue will entail establishing an upper bound for the generic generator degree of first syzygy modules (Proposition 3.2), as well as estimating the initial degree of certain colon ideals (Propositions 3.5 and 3.6).

We begin by recalling a result from [2], which in turn uses earlier work on the 'fundamental class' (see [5, p.34], [12, 4.11 and 5.13], [13, 3.1]). 
Proposition 3.1 ([2, 1.1]). Let $k$ be a perfect field and let $R$ be a standard graded $k$-algebra of dimension $d$ such that $R_{\mathfrak{p}}$ is regular for every minimal prime $\mathfrak{p}$ of dimension d. Write $\omega=\omega_{R}$ and let $C$ be defined by the exact sequence

$$
0 \longrightarrow[\omega]_{\leq d} R \stackrel{\text { nat }}{\longrightarrow} \omega \longrightarrow C \longrightarrow 0 .
$$

Then $\operatorname{Supp}(C) \subset \operatorname{Sing}(R)$.

Proposition 3.2. Let $k$ be a perfect field and $S$ a standard graded Gorenstein $k$ algebra of dimension $D$. Let $I$ be an ideal of height $g$ such that $I_{\mathfrak{P}}$ is prime for every prime ideal $\mathfrak{P}$ of height $g$ containing $I$. Assume $I$ is generated by $m \geq g+1$ forms $f_{1}, \ldots, f_{m}$ of degrees $\delta_{1} \geq \ldots \geq \delta_{m}$, and write $\Delta=\sum_{i=1}^{g+1} \delta_{i}+D-g+a(S)$. Let $H_{1}$ be the first Koszul homology of the elements $f_{1}, \ldots, f_{m}$ and $C$ the module defined by the exact sequence

$$
0 \longrightarrow\left[H_{1}\right]_{\leq \Delta} S \stackrel{\text { nat }}{\longrightarrow} H_{1} \longrightarrow C \longrightarrow 0 .
$$

Then $C_{\mathfrak{P}}=0$ for every prime ideal $\mathfrak{P}$ of $S$ such that $I_{\mathfrak{P}}$ is a complete intersection and $S_{\mathfrak{P}} / I_{\mathfrak{P}}$ is regular. If either $g \geq 2$ and $\delta_{3} \geq 2$ or $g \geq 1$ and $S$ is not a polynomial ring, one can replace $H_{1}$ by the first syzygy module of the elements $f_{1}, \ldots, f_{m}$.

Proof. Fix a prime $\mathfrak{P}$ so that $I_{\mathfrak{P}}$ is a complete intersection and $S_{\mathfrak{P}} / I_{\mathfrak{P}}$ is regular. We may assume that $k$ is infinite and that $f_{1}, \ldots, f_{g}$ form a regular sequence generating $I_{\mathfrak{P}}$. Notice that

$$
\left(\left(f_{1}, \ldots, f_{g}\right): I\right) /\left(f_{1}, \ldots, f_{g}\right) \cong \omega_{S / I}\left(-\sum_{i=1}^{g} \delta_{i}-a(S)\right) .
$$

Therefore Proposition 3.1 shows that there exists a homogeneous element $\beta$ of degree $D-g+\sum_{i=1}^{g} \delta_{i}+a(S)$ in $\left(f_{1}, \ldots, f_{g}\right): I$ generating the factor module $\left(\left(f_{1}, \ldots, f_{g}\right): I\right) /\left(f_{1}, \ldots, f_{g}\right)$ locally at $\mathfrak{P}$. In particular, $\beta \notin \mathfrak{P}$ because $\left(\left(f_{1}, \ldots, f_{g}\right): I\right)_{\mathfrak{P}}=S_{\mathfrak{P}}$. Hence for $g+1 \leq j \leq m$, there exist homogeneous syzygies

$$
\beta f_{j}-\sum_{i=1}^{g} \lambda_{i j} f_{i}=0
$$

of $f_{1}, \ldots, f_{m}$ that have degree at most $\Delta$ and whose images generate $H_{1}$ locally at $\mathfrak{P}$.

Finally, if either $g \geq 2$ and $\delta_{3} \geq 2$ or $g \geq 1$ and $S$ is not a polynomial ring, then the Koszul relations among $f_{1}, \ldots, f_{m}$ have degrees at most $\Delta$.

We are now going to introduce the $\alpha$-invariant of a graded ring that will be the basis for many estimates proved in this section.

Definition 3.3. Let $k$ be a field and $R$ a standard graded $k$-algebra of dimension d. We define

$$
\alpha(R)=\min \{a(S)\} \in \mathbb{Z} \cup\{\infty\},
$$

where $S$ ranges over all standard graded Gorenstein $k$-algebras of dimension $d$ mapping homogeneously onto $R$ such that $S_{\mathfrak{P}}=R_{\mathfrak{P}}$ for every minimal prime $\mathfrak{P} \in \operatorname{Supp}_{S}(R)$ of dimension $d$. 
Remark 3.4.

(a) One has

$$
a(R) \leq \alpha(R),
$$

because $\omega_{R} \hookrightarrow \omega_{S}$ for every $S$ as in Definition 3.3 .

(b) Write $R=k\left[X_{1}, \ldots, X_{n}\right] / I$ as a factor ring of a polynomial ring, where $I$ is an ideal of height $g$ generated by forms of degrees $\delta_{1} \geq \ldots \geq \delta_{m}$. If $\beta_{1}, \ldots, \beta_{g}$ is a homogeneous regular sequence contained in $I$ that generates $I$ at each of its minimal primes of height $g$, then

$$
\alpha(R) \leq \sum_{i=1}^{g} \operatorname{deg}\left(\beta_{i}\right)-n .
$$

In particular, whenever $k$ is infinite and $I$ is generically a complete intersection, we have

$$
\alpha(R) \leq \sum_{i=1}^{g} \delta_{i}-n
$$

Proposition 3.5. Let $k$ be an infinite perfect field and $R$ a standard graded $k$ algebra of dimension $d$. Let $H$ be a homogeneous $R$-ideal such that $\operatorname{dim} R / H=d$ and $R_{\mathfrak{p}}$ is regular for every minimal prime $\mathfrak{p}$ of dimension d containing $H$. If $\alpha(R)$ is finite, there exists a homogeneous element of degree $\alpha(R)+d$ in $0: H$ that is not contained in any minimal prime $\mathfrak{p}$ of dimension d containing $H$.

Proof. By $\mathfrak{p}_{1}, \ldots, \mathfrak{p}_{s}$ we denote the minimal primes of dimension $d$ containing $H$. Let $S$ be as in Definition 3.3 so that $\alpha(R)=a(S)$. Write $N$ and $\mathfrak{P}_{i}$ for the preimages of $H$ and $\mathfrak{p}_{i}$ in $S$. Notice that $H=N R$, that $N_{\mathfrak{P}_{i}}=0$, and that $(S / N)_{\mathfrak{P}_{i}}=(R / H)_{\mathfrak{p}_{i}}$ is regular for every $\mathfrak{P}_{i}$.

The beginning of the proof of Proposition [3.2, with $g=0$, shows that there exists a homogeneous element $\beta \in 0:_{S} N$ of degree $a(S)+d$ with $\beta \notin \mathfrak{P}_{i}$ for each of the finitely many primes $\mathfrak{P}_{i}$. Thus, denoting the image of $\beta$ in $R$ by $\gamma$ we obtain $\gamma \in\left(0:_{S} N\right) R \subset 0:_{R} N R=0:_{R} H$ and $\gamma \notin \mathfrak{p}_{i}$ for each $\mathfrak{p}_{i}$.

The next proposition shows that the $\alpha$-invariant can be replaced by $a(R)$ in the above estimate if $R$ has dimension one. We will use the notation $H^{\text {unm }}$ for the unmixed part of an ideal $H$, which is the intersection of the primary components of maximal dimension.

Proposition 3.6. Let $k$ be an infinite field and $R$ a standard graded CohenMacaulay k-algebra of dimension 1 . Let $H$ be a homogeneous $R$-ideal such that $H_{\mathfrak{p}}=0$ for every minimal prime $\mathfrak{p}$ of $H$. Then there exists a homogeneous element of degree $a(R)+1$ in $0: H$ that is not contained in any minimal prime $\mathfrak{p}$ of $H$.

Proof. Write $\mathfrak{m}$ for the homogeneous maximal ideal of $R$ and set $L=0: H$. Notice that $0: H^{\mathrm{unm}}=0: H$ since $R$ is Cohen-Macaulay. Passing to the unmixed part of $H$ we can suppose that $R / H$ is Cohen-Macaulay of dimension 1 . Furthermore $R / L$ is either zero or Cohen-Macaulay of dimension 1 , and $R / H+L$ has finite length. Now the short exact sequence

$$
0 \longrightarrow R / H \cap L \longrightarrow R / H \oplus R / L \longrightarrow R / H+L \longrightarrow 0
$$

induces an exact sequence of local cohomology modules

$$
0 \longrightarrow H_{\mathfrak{m}}^{0}(R / H+L)=R / H+L \longrightarrow H_{\mathfrak{m}}^{1}(R / H \cap L) \text {. }
$$


As $a(R / H \cap L) \leq a(R)$ we conclude that $R / H+L$ is concentrated in degrees $\leq a=a(R)$. Hence $\mathfrak{m}^{a+1} \subset H+L$. Thus any homogeneous non-zero-divisor of degree $a+1$ can be written in the form $h+l$, where $h, l$ are homogeneous elements of degree $a+1$ in $H, L$ respectively. Now $l$ is an element with the desired properties.

With the next two corollaries we answer the question raised at the beginning of the section. We give a bound for the initial degrees of annihilators of submodules generated by graded components of $\omega$ and we estimate how large the integer $i$ has to be chosen in Corollary 2.9)(c).

Theorem 3.7. Let $k$ be an infinite perfect field, let $R$ be a standard graded reduced equidimensional $k$-algebra of dimension $d$ with $\omega=\omega_{R}$, and let $t$ be an integer. If $[\omega]_{t} R$ is not a faithful $R$-module, then

$$
\operatorname{indeg}\left(\operatorname{ann}_{R}\left([\omega]_{t} R\right)\right) \leq \alpha(R)+d .
$$

Proof. We may assume that $\alpha(R)$ is finite. Since $R$ is generically Gorenstein, $\omega$ is isomorphic to a suitable shift of a homogeneous $R$-ideal $W$, say $\omega \cong W(s)$. As $[W]_{t+s} R$ is not faithful it is contained in some minimal prime $\mathfrak{p}$ of $R$. Now

$$
\operatorname{ann}_{R}\left([\omega]_{t} R\right)=\operatorname{ann}_{R}\left([W]_{t+s} R\right) \supset 0:_{R} \mathfrak{p},
$$

and the latter ideal contains a nonzero homogeneous element of degree $\alpha(R)+d$ according to Proposition 3.5

Replacing Proposition 3.5 by Proposition 3.6 in the proof of Theorem 3.7yields a better estimate for the initial degree in the one-dimensional case that is still weaker though than the one implied by Corollary 2.8 .

Corollary 3.8. Let $k$ be an infinite perfect field, let $R$ be a standard graded reduced Cohen-Macaulay $k$-algebra of dimension $d \geq 1$ with homogeneous maximal ideal $\mathfrak{m}$, and let $t$ be an integer. Write $\omega=\omega_{R}$ and $a=a(R)$. Then $[\omega]_{t} R$ is a faithful $R$-module if and only if $J^{i+a+t}: \mathfrak{m}^{a+d} \subset \mathfrak{m}^{i}$ for some $i \geq \alpha(R)+d+1$.

Proof. The forward direction follows from Corollary 2.9 and the converse from Theorems 2.7 and 3.7.

Under suitable additional assumptions the bound for $i$ in Corollary 3.8 can be improved, most notably by replacing the invariant $\alpha(R)$ by the more traditional $a(R)$. This is the content of the next three results of the section.

Proposition 3.9. Let $k$ be a field, let $R$ be a standard graded Cohen-Macaulay $k$-algebra of dimension $d \geq 1$ with homogeneous maximal $i d e a l ~ \mathfrak{m}$, and let $t$ be an integer. Write $\omega=\omega_{R}, a=a(R)$, and $-^{\vee}=\operatorname{Hom}_{R}(-, \omega)$. If $\omega /\left(\left([\omega]_{\leq t} R\right)^{\vee \vee}\right)$ is Cohen-Macaulay, then $\operatorname{ann}_{R}\left([\omega]_{t} R\right)$ is a Cohen-Macaulay module with CastelnuovoMumford regularity $\leq d-t-1$. In particular, whenever $t \geq-a$ one has that $[\omega]_{t} R$ is a faithful $R$-module if and only if $J^{i+a+t}: \mathfrak{m}^{a+d} \subset \mathfrak{m}^{i}$ for some $i \geq a+d$.

Proof. In light of Corollary 2.9, the second statement follows from the first claim and Theorem 2.7. Indeed, the bound on the Castelnuovo-Mumford regularity shows that $\operatorname{ann}_{R}\left([\omega]_{t} R\right)$ is generated in degrees $\leq d-t-1 \leq a+d-1$. Hence this annihilator vanishes by Theorem 2.7 if $J^{i+a+t}: \mathfrak{m}^{a+d} \subset \mathfrak{m}^{i}$ for some $i \geq a+d$. 
Thus it suffices to prove the assertion about $\operatorname{ann}_{R}\left([\omega]_{t} R\right)$. We may assume that this annihilator is not zero. One has

$$
\operatorname{ann}_{R}\left([\omega]_{t} R\right)=\operatorname{ann}_{R}\left([\omega]_{\leq t} R\right)=\operatorname{ann}_{R}\left(\left([\omega]_{\leq t} R\right)^{\vee \vee}\right) .
$$

The first equality is part of Theorem 2.7. To see the second equality notice that since $\omega$ is a maximal Cohen-Macaulay $R$-module, the annihilator of every submodule is either the unit ideal or an unmixed ideal of height zero. In particular, two submodules of $\omega$ have the same annihilator if they coincide locally at every minimal prime of $R$. Since $[\omega]_{\leq t} R$ and $\left([\omega]_{\leq t} R\right)^{\vee \vee}$ are generically equal, the second equality now follows. Thus we may restrict our attention to the annihilator ideal $\operatorname{ann}_{R}\left(\left([\omega]_{\leq t} R\right)^{\vee \vee}\right)$. Likewise, since $\operatorname{ann}_{R}\left(\left([\omega]_{t} R\right)^{\vee \vee}\right) \neq 0=\operatorname{ann}_{R}(\omega)$, it follows that $\left([\omega]_{\leq t} R\right)^{\vee \vee}$ and $\omega$ cannot coincide locally at every minimal prime of $R$. Thus $\omega /\left(\left([\omega]_{\leq t} R\right)^{\vee \vee}\right)$ has dimension $d$, hence is a maximal Cohen-Macaulay $R$-module.

Write $R=S / I$ with $S=k\left[X_{1}, \ldots, X_{n}\right]$ a polynomial ring and $I$ a homogeneous $S$-ideal of height $g$. Choose a regular sequence $\beta=\beta_{1}, \ldots, \beta_{g}$ of forms of degree $\delta \gg 0$ contained in $I$ and set $L=(\beta): S I$. Notice that $S / L$ is a $d$-dimensional Cohen-Macaulay ring. Moreover one has $(L /(\beta))(g \delta-n) \cong \omega$. Thus there exists a homogeneous ideal $H$ of $S$ so that $(\underline{\beta}) \subset H \subset \bar{L}$ and $(H /(\underline{\beta}))(g \delta-n) \cong\left([\omega]_{\leq t} R\right)^{\vee \vee}$. Clearly

$$
\operatorname{ann}_{R}\left(\left([\omega]_{\leq t} R\right)^{\vee \vee}\right)=\left((\underline{\beta}):_{S} H\right) / I .
$$

Since $L / H$ is isomorphic to a shift of the module $\omega /\left(\left([\omega]_{\leq t} R\right)^{\vee \vee}\right)$ it follows that $L / H$ is a maximal Cohen-Macaulay $R$-module. Therefore $S / H$ is a $d$-dimensional Cohen-Macaulay ring, and hence so is $S /\left((\beta):_{S} H\right)$. Thus $\left((\beta):_{S} H\right) / I$ is a maximal Cohen-Macaulay $R$-module, proving the assertion in the proposition about the Cohen-Macaulayness of the annihilator ideal.

Applying $\operatorname{Ext}_{S}^{g}(-, S(-n))$ to the exact sequence

$$
0 \longrightarrow\left((\underline{\beta}):_{S} H\right) / I \longrightarrow S / I \longrightarrow S /\left((\underline{\beta}):_{S} H\right) \longrightarrow 0
$$

of maximal Cohen-Macaulay $R$-modules we obtain this exact sequence

$$
0 \longrightarrow \omega_{S /((\underline{\beta}): H)} \longrightarrow \omega_{S / I}=\omega \longrightarrow \operatorname{Ext}_{S}^{g}\left(\left((\underline{\beta}):_{S} H\right) / I, S(-n)\right) \longrightarrow 0 .
$$

The canonical module on the left satisfies

$$
\omega_{S /((\underline{\beta}): H)} \cong(H /(\underline{\beta}))(g \delta-n) \cong\left([\omega]_{\leq t} R\right)^{\vee \vee} \supset[\omega]_{\leq t} R,
$$

where the first isomorphism holds because $H$ is unmixed. Therefore the above exact sequence shows that $\operatorname{Ext}_{S}^{g}\left(\left((\beta):_{S} H\right) / I, S(-n)\right)$ is concentrated in degrees $\geq t+1$, and hence by local duality the local cohomology module $H_{\mathfrak{m}}^{d}\left(\left((\beta):_{S} H\right) / I\right)$ is concentrated in degrees $\leq-t-1$. As $\left((\beta):_{S} H\right) / I$ is a $d$-dimensional CohenMacaulay module we deduce that it has regularity $\leq d-t-1$.

We follow suit with an estimate for rings of type 2 that does not require the Cohen-Macaulayness of $\omega /\left(\left([\omega]_{\leq t} R\right)^{\vee \vee}\right)$.

Proposition 3.10. Let $k$ be a field and $R$ a standard graded geometrically reduced Cohen-Macaulay $k$-algebra of type 2 and dimension $d \geq 1$ with homogeneous maximal ideal $\mathfrak{m}$. Write $R=S / I$ with $S=k\left[X_{1}, \ldots, X_{n}\right]$ a polynomial ring and $I$ a homogeneous $S$-ideal of height $g$. Consider the last map in a minimal homogeneous 
free $S$-resolution of $R$,

$$
0 \longrightarrow S\left(-l_{1}\right) \oplus S\left(-l_{2}\right) \stackrel{\varphi}{\longrightarrow} \bigoplus_{i=1}^{m} S\left(-k_{i}\right)
$$

where $l_{1} \leq l_{2}$ and $k_{1} \leq \ldots \leq k_{m}$. Write $\omega=\omega_{R}$ and $a=a(R)$. If $[\omega]_{-a} R$ is not $a$ faithful $R$-module, then

$$
\operatorname{indeg}\left(\operatorname{ann}_{R}\left([\omega]_{-a} R\right)\right) \leq g l_{1}+l_{2}-\sum_{i=1}^{g+1} k_{i}-g=a+d+g l_{1}-\sum_{i=1}^{g+1} k_{i} .
$$

Equivalently, $[\omega]_{-a} R$ is a faithful $R$-module if and only if $J^{i}: \mathfrak{m}^{a+d}=\mathfrak{m}^{i}$ for some $i \geq a+d+g l_{1}-\sum_{i=1}^{g+1} k_{i}+1$.

Proof. By Theorem 2.7 and Corollary 2.10 it suffices to prove the first statement. We may assume that the field $k$ is infinite and perfect. Since $R$ is Cohen-Macaulay we obtain the following presentation:

$$
\bigoplus_{i=1}^{m} S\left(k_{i}\right) \stackrel{\varphi^{*}}{\longrightarrow} S\left(l_{1}\right) \oplus S\left(l_{2}\right) \longrightarrow \omega(n) \longrightarrow 0 .
$$

Using the standard bases of the free modules this presentation gives rise to homogeneous generators $w_{1}, w_{2}$ of $\omega(n)$ and a matrix

$$
\left(\begin{array}{lll}
f_{1} & \cdots & f_{m} \\
h_{1} & \cdots & h_{m}
\end{array}\right)
$$

representing $\varphi^{*}$. Notice that $\operatorname{deg}\left(w_{1}\right)=-l_{1} \geq \operatorname{deg}\left(w_{2}\right)=-l_{2}, \operatorname{deg}\left(f_{i}\right)=l_{1}-k_{i}$, and $\operatorname{deg}\left(h_{i}\right)=l_{2}-k_{i}$. One has $\operatorname{deg}\left(w_{1}\right)>\operatorname{deg}\left(w_{2}\right)$ since otherwise $[\omega]_{-a} R=\omega$ would be faithful. Therefore

$$
\operatorname{ann}_{R}\left([\omega]_{-a} R\right)=\operatorname{ann}_{R} w_{2} .
$$

Further observe that

$$
w_{2} R:_{S} w_{1}=\left(f_{1}, \ldots, f_{m}\right)
$$

and

$$
\operatorname{ann}_{S} w_{2}=\left\{\sum \lambda_{i} h_{i} \mid \sum \lambda_{i} f_{i}=0\right\}
$$

the last equality obtains because an element $\varepsilon$ of $S$ belongs to $\operatorname{ann}_{S} w_{2}$ if and only if the vector $\left(\begin{array}{c}0 \\ \varepsilon\end{array}\right)$ is in the column space of the above matrix.

As $\operatorname{ann}_{R} w_{2} \neq 0$ and $R$ is unmixed, there exists a minimal prime $\mathfrak{p}$ of $R$ such that $\left(\operatorname{ann}_{R} w_{2}\right)_{\mathfrak{p}} \neq 0$. In particular, $w_{2} R_{\mathfrak{p}} \neq w_{1} R_{\mathfrak{p}}+w_{2} R_{\mathfrak{p}}$, because the latter module is faithful. Thus the ideal $\left(f_{1}, \ldots, f_{m}\right)=w_{2} R:_{S} w_{1}$ is contained in $\mathfrak{P}$, the preimage of $\mathfrak{p}$ in $S$. On the other hand, this ideal contains $I$. It follows that $\left(f_{1}, \ldots, f_{m}\right)$ has height $g$. Furthermore, since $I$ is radical the localization $\left(f_{1}, \ldots, f_{m}\right) S_{\mathfrak{Q}}$ is a complete intersection prime ideal for every prime $\mathfrak{Q}$ of height $g$ in $S$ containing $\left(f_{1}, \ldots, f_{m}\right)$. Finally, $g=\mathrm{ht} I=\mathrm{ht} I_{2}(\varphi) \leq m-1$; the second equality follows from the Cohen-Macaulayness of $R$ and the inequality holds by the Eagon-Northcott bound on the height of determinantal ideals. Now Proposition 3.2 shows that locally at $\mathfrak{P}$, the syzygy module of $\left(f_{1}, \ldots, f_{m}\right)$ is generated by its elements of degrees $\leq \sum_{i=1}^{g+1}\left(l_{1}-k_{i}\right)+n-g+a(S)$ and by the Koszul relations. Therefore (3.1) gives

$$
\left(\operatorname{ann}_{S} w_{2}\right)_{\mathfrak{P}}=\left(\left[\operatorname{ann}_{S} w_{2}\right]_{\leq \sum_{i=1}^{g+1}\left(l_{1}-k_{i}\right)+n-g+a(S)+l_{2}-l_{1}}+I_{2}\left(\varphi^{*}\right)\right)_{\mathfrak{P}}
$$


As $I_{2}\left(\varphi^{*}\right) \subset \operatorname{ann}_{S} \omega=I$ we conclude that

$$
\begin{aligned}
\left(\operatorname{ann}_{R} w_{2}\right)_{\mathfrak{p}} & =\left(\left[\operatorname{ann}_{R} w_{2}\right]_{\leq \sum_{i=1}^{g+1}\left(l_{1}-k_{i}\right)+n-g+a(S)+l_{2}-l_{1}}\right)_{\mathfrak{p}} \\
& =\left(\left[\operatorname{ann}_{R} w_{2}\right]_{\leq g l_{1}+l_{2}-\sum_{i=1}^{g+1} k_{i}-g}\right)_{\mathfrak{p}} .
\end{aligned}
$$

The assertion now follows since $\left(\operatorname{ann}_{R} w_{2}\right)_{\mathfrak{p}} \neq 0$.

Corollary 3.11. Let $k$ be a field and let $R$ be a standard graded equidimensional geometrically reduced $k$-algebra of dimension $d \geq 1$ with homogeneous maximal ideal $\mathfrak{m}$. Assume that $R$ is an almost complete intersection of embedding codimension 2 . Write $\omega=\omega_{R}$ and $a=a(R)$. If $[\omega]_{-a} R$ is not a faithful $R$-module, then

$$
\operatorname{indeg}\left(\operatorname{ann}_{R}\left([\omega]_{-a} R\right)\right) \leq a+d .
$$

Equivalently, $[\omega]_{-a} R$ is a faithful $R$-module if and only if $J^{i}: \mathfrak{m}^{a+d}=\mathfrak{m}^{i}$ for some $i \geq a+d+1$.

Proof. Notice that $R$ is Cohen-Macaulay by the Syzygy Theorem and has type 2; see [6, 2.1]. Hence we may apply Proposition [3.10 with $g=2$. It suffices to show that $2 l_{1}-\sum_{i=1}^{3} k_{i} \leq 0$. This holds, because $k_{3}=l_{1}+l_{2}-k_{1}-k_{2}$ by the Hilbert-Burch Theorem and $l_{1} \leq l_{2}$.

We finish this section with a different estimate for initial degrees of annihilators: an estimate from below.

Proposition 3.12. Let $k$ be a field, let $R$ be a standard graded Cohen-Macaulay $k$-algebra of dimension $d$, and let $H$ be a homogeneous $R$-ideal. Write $c=c(R)$ as in 2.1. One has indeg $(0: H) \geq c+d+1-e(R / H)$.

Proof. We may assume that $k$ is infinite and that $0: H \neq 0$. Set $e=e(R / H)$. We prove the claim by induction on $d$. First let $d=0$. In this case, $e=\lambda(R / H)$. Therefore $\mathfrak{m}^{e} \subset H$, which gives $0: H \subset 0: \mathfrak{m}^{e}$. But indeg $\left(0: \mathfrak{m}^{e}\right) \geq c+1-e$, because $c$ is the initial degree of the socle of $R$.

Next let $d \geq 1$. Notice that $0: H^{\text {unm }}=0: H$ since $R$ is Cohen-Macaulay and that $e\left(R / H^{\text {unm }}\right)=e(R / H)$ by the associativity formula for the multiplicity. Hence we may replace $H$ by $H^{\text {unm }}$ to assume that $H$ is unmixed. Since $0: H \neq 0$ we have ht $H=0$. Furthermore, $0: H$ is unmixed with ht $(0: H)=0$ or else $0: H=R$. Now let $x \in R$ be a linear form that is regular on $R$, and write for images in the $(d-1)$-dimensional Cohen-Macaulay ring $\bar{R}=R /(x)$. Notice that $x$ is regular modulo $H$ and is regular modulo $0: H$ unless $0: H=R$. It follows that $e(\bar{R} / \bar{H})=e(R / H)$ and indeg $\left(\overline{0:_{R} H}\right)=\operatorname{indeg}\left(0:_{R} H\right)$. Furthermore, $c(\bar{R})=c(R)+1$ as $\omega_{\bar{R}} \cong \omega_{R}(1)$. Thus we conclude

$$
\begin{aligned}
\operatorname{indeg}\left(0:_{R} H\right) & =\operatorname{indeg}\left(\overline{0:_{R} H}\right) \\
& \geq \operatorname{indeg}(\overline{0}: \bar{R} \bar{H}) \\
& \geq c(\bar{R})+\operatorname{dim} \bar{R}+1-e(\bar{R} / \bar{H}) \\
& =(c+1)+(d-1)+1-e \\
& =c+d+1-e .
\end{aligned}
$$

since $\overline{0:_{R} H} \subset \overline{0}: \bar{R} \bar{H}$

by induction hypothesis

If $R$ is zero-dimensional in the above proposition, then the lower bound $c+1-$ $e(R / H)$ can be improved to $c-a(R / H)$. According to (2.2), this sharper estimate immediately gives the inclusion $J^{i}: \mathfrak{m}^{j} \subset \mathfrak{m}^{i-j+c+d}$ in the setting of Corollary 2.17 


\section{THE CORE OF STANDARD GRADED ALGEBRAS}

In this section we apply the previous results to the core of powers of the maximal ideal.

Assumptions 4.1. In addition to the assumptions of 2.1, suppose that if char $k>$ 0 , then $k$ is infinite and $R$ is geometrically reduced.

The next result is essentially present, in a more general form, in [7, 4.2].

Theorem 4.2. With assumptions as in 4.1, one has for every $n \geq 1$,

$$
\operatorname{core}\left(\mathfrak{m}^{n}\right)=J^{n d+a+1}: \mathfrak{m}^{a+d} .
$$

Proof. From [15, 2.3 and 2.5] we know that

$$
\operatorname{core}\left(\mathfrak{m}^{n}\right)=\left(J^{[n]}\right)^{j+1}:\left(\mathfrak{m}^{n}\right)^{j} \text { for } j \gg 0 .
$$

Furthermore

$$
\begin{aligned}
\left(J^{[n]}\right)^{j+1}:\left(\mathfrak{m}^{n}\right)^{j} & =\left(J^{[n]}\right)^{[j+1]}:\left(\mathfrak{m}^{n}\right)^{j d} & & \text { by (2.2) } \\
& =J^{[n j+n]}: \mathfrak{m}^{n j d} & & \\
& =J^{n j+n}: \mathfrak{m}^{n j+n-n d+d-1} & & \text { by (2.2) } \\
& =J^{n d+a+1}: \mathfrak{m}^{a+d} & & \text { by Remark 2.2. }
\end{aligned}
$$

The above theorem relates the core of powers of the maximal ideal to the colon ideals studied in the previous sections. We leave it to the reader to express most of the earlier results in terms of cores. Here we only collect the main applications:

Corollary 4.3. With assumptions as in 4.1, one has for every $n \geq 1$ :

(a) core $\left(\mathfrak{m}^{n}\right)=\mathfrak{m}^{n d+a+1}+N$ for some ideal $N$ of height zero;

(b) $\mathfrak{m}^{n d+a+1} \subset \operatorname{core}\left(\mathfrak{m}^{n}\right) \subset \mathfrak{m}^{n d+b+1}$.

Proof. The result follows immediately from Theorem 4.2 and Corollaries 2.15 and 2.17 .

Item (b) of the next theorem was asserted in [11, 4.1], assuming only that $R$ is reduced. However, as the theorem shows, this statement is equivalent to the faithfulness of the module $[\omega]_{-a} R$. Furthermore, $R$ being geometrically reduced is essential according to [7, 5.1].

Theorem 4.4. With assumptions as in 4.1, the following are equivalent:

(a) $[\omega]_{-a} R$ is faithful;

(b) $\operatorname{core}\left(\mathfrak{m}^{n}\right)=\mathfrak{m}^{n d+a+1}$ for every $n \geq 1$;

(c) core $\left(\mathfrak{m}^{n}\right)$ is generated in one degree for some $n \gg 0$.

Proof. Theorem 4.2 and Corollary 2.10 show that (a) implies (b). If (c) holds, then according to Theorem 4.2 and Corollary 2.16 one has $J^{n d+a+1}: \mathfrak{m}^{a+d}=\mathfrak{m}^{n d+a+1}$. Notice that $n d+a+1 \gg 0$. Thus again Corollary 2.10 gives that (a) obtains.

Corollary 4.5. In addition to the assumptions of 4.1, suppose that one of the following conditions holds :

- $R$ is reduced and $[\omega]_{\geq d+1}=\operatorname{core}(\mathfrak{m}) \omega$;

- $d=1$;

- $R$ is a reduced almost complete intersection of embedding codimension 2. 
Then the following are equivalent:

(a) $[\omega]_{-a} R$ is faithful;

(b) $\operatorname{core}(\mathfrak{m})=\mathfrak{m}^{a+d+1}$;

(c) core $(\mathfrak{m})$ is generated in one degree.

Proof. We apply Theorem 4.2 and Corollary 2.16 . In the first case we also use Corollary 2.10 via Theorem 2.3(a), in the second case Corollary 2.11, and in the third case Corollary 3.11 .

\section{THE CORE OF POINTS}

Assumptions and Discussion 5.1. Let $k$ be an infinite field and let $X=\left\{P_{1}, \ldots\right.$, $\left.P_{s}\right\}$ be a set of $s$ reduced rational points in $\mathbb{P}_{k}^{n}$. Write $S=k\left[x_{0}, \ldots, x_{n}\right]$ for the polynomial ring and $R=S / I_{X}$ for the homogeneous coordinate ring of $X \subset \mathbb{P}_{k}^{n}$. Let $\mathfrak{m}$ denote the homogeneous maximal ideal of $R, K$ its total ring of quotients, $B$ the integral closure of $R$ in $K$, and $\mathcal{C}=R:_{K} B$ the conductor. Furthermore, we write $\omega=\omega_{R}, a=a(R), b=b(R)$ and we define core $(X)=$ core $(\mathfrak{m})$. Finally, let $y \in R$ be a linear form that is $R$-regular and set $J=y R$. Notice that $a \leq s-2$ and that $R$ is geometrically reduced.

Homogeneous polynomials $f_{1}, \ldots, f_{s}$ in $S$ are called separators of $X$ if $f_{i}\left(P_{j}\right)=$ $\delta_{i j}$ for every $i, j$. They are called minimal separators if in addition each $f_{i}$ has smallest possible degree.

With the next lemma we recall a known fact describing the conductor in terms of minimal separators (see for instance [8,3.13]). We include a proof for the convenience of the reader.

Lemma 5.2. With assumptions as in 5.1, let $h_{1}, \ldots, h_{s}$ be a collection of separators of $X$ and $f_{1}, \ldots, f_{s}$ a collection of minimal separators. One has $h_{i} R \subset$ $f_{i} R$ and $f_{1}, \ldots, f_{s}$ minimally generate $\mathcal{C}$ as an $R$-ideal; in particular, $\mathfrak{m}^{a+1} \subset$ $\left(f_{1}, \ldots, f_{s}\right) R=\mathcal{C}$ and $\operatorname{deg}\left(f_{i}\right) \leq a+1$.

Proof. Fix $\lambda_{i j} \in k$ with $P_{i}=\left(\lambda_{i_{0}}: \ldots: \lambda_{i_{n}}\right)$. We use these identifications of nonnegatively graded rings,

$$
\varphi: R \hookrightarrow B \cong k[t] \times \ldots \times k[t]=\bigoplus_{i=1}^{s} k[t] e_{i},
$$

where $k[t]$ is a standard graded polynomial ring, $e_{i}$ are standard basis elements of degree zero, and $\varphi$ maps the image in $R$ of a polynomial $h \in[S]_{l}$ to the tuple $\sum h\left(\lambda_{i_{0}}, \ldots, \lambda_{i_{n}}\right) t^{l} e_{i}$. Thus the elements $t^{l_{1}} e_{1}, \ldots, t^{l_{s}} e_{s}$ belong to $R$ if and only if there exist separators $h_{1}, \ldots, h_{s}$ of degrees $l_{1}, \ldots, l_{s}$, in which case $t^{l_{i}} e_{i} R=h_{i} R$. From this we see that $h_{i} R \subset f_{i} R$. It also follows that the minimal separators $f_{1}, \ldots, f_{s}$ minimally generate the largest $R$-ideal of the form $\sum t^{l_{i}} e_{i} R=\sum t^{l_{i}} k[t] e_{i}$, equivalently, the largest homogeneous $B$-ideal contained in $R$. However, this ideal is the conductor $\mathcal{C}$.

Finally, the long exact sequence of local cohomology shows that $B / R$ is concentrated in degrees $\leq a$; hence $\mathfrak{m}^{a+1} \subset \mathcal{C}$.

The next proposition gives a geometric interpretation of the core of points in terms of separators. 
Proposition 5.3. With assumptions as in 5.1, let $f_{1}, \ldots, f_{s}$ be minimal separators of $X$. One has

(a) $\operatorname{core}(X)=y \mathcal{C}=\mathfrak{m} \mathcal{C}=y R\left(f_{1}, \ldots, f_{s}\right)=\mathfrak{m}\left(f_{1}, \ldots, f_{s}\right)$;

(b) $[\omega]_{\geq 2}=\operatorname{core}(X) \omega$.

Proof. According to Theorem 4.2 one has $\operatorname{core}(X)=J^{a+2}: \mathfrak{m}^{a+1}$. Now the first and second equality in (a) follow from Remark 2.19, and (b) is a consequence of Proposition 2.6. Finally, Lemma 5.2 implies $\mathcal{C}=\left(f_{1}, \ldots, f_{s}\right) R$.

Corollary 5.4. In addition to the assumptions of 5.1 suppose that $X=Y \cup Z$, where $Y$ is contained in a hypersurface $f=0$ and $Z$ is a collection of e reduced points whose homogeneous coordinate ring has a-invariant $a^{\prime}$. One has

$$
\mathfrak{m}^{a+2}+f \mathfrak{m}^{e} \subset \mathfrak{m}^{a+2}+f \mathfrak{m}^{a^{\prime}+2} \subset \operatorname{core}(X) \subset \mathfrak{m}^{b+2} .
$$

Proof. Let $h_{1}, \ldots, h_{e}$ be minimal separators of $Z$, and write $H$ for the defining ideal of $Z$ in $X$. From Lemma 5.2 we know that $\mathfrak{m}^{a^{\prime}+1} \subset\left(h_{1}, \ldots, h_{e}\right) R+H$. Since $f H=0$ in $R$, multiplying this equation by $f$ we obtain $f \mathfrak{m}^{a^{\prime}+1} \subset\left(f h_{1}, \ldots, f h_{e}\right) R$. However, those elements of $f h_{1}, \ldots, f h_{e}$ that are not contained in $I_{X}$ form part of a collection of separators of $X$. Hence $\left(f h_{1}, \ldots, f h_{e}\right) R \subset \mathcal{C}$ by the same Lemma 5.2 , and therefore $f \mathfrak{m}^{a^{\prime}+2} \subset \operatorname{core}(X)$ according to Proposition 5.3(a). The remaining assertions follow from Discussion 5.1 and Corollary 4.3(b).

The previous result suggests that the shape of the core is related to uniformity properties of the set of points. We recall one such condition: The scheme $X \subset$ $\mathbb{P}_{k}^{n}$ is said to have the Cayley-Bacharach property if each subscheme of the form $X \backslash\left\{P_{i}\right\} \subset \mathbb{P}_{k}^{n}$ has the same Hilbert function.

Corollary 5.5. With assumptions as in 5.1, the scheme $X$ has the CayleyBacharach property if and only if $\operatorname{core}(X)=\mathfrak{m}^{a+2}$.

Proof. It is easy to see that $X$ has the Cayley-Bacharach property if and only if the minimal separators all have the same degree. According to Proposition 5.3(a), this means that $\operatorname{core}(X)$ is generated in one degree, which in turn is equivalent to $\operatorname{core}(X)=\mathfrak{m}^{a+2}$ as shown in Corollary 4.5.

Alternatively, in [8, 3.5] the Cayley-Bacharach property has been characterized in terms of the faithfulness of $[\omega]_{-a} R$. Again according to Corollary 4.5, the latter condition holds if and only if $\operatorname{core}(X)=\mathfrak{m}^{a+2}$.

The next example illustrates the previous two corollaries.

Example 5.6. In addition to the assumptions of 5.1 suppose char $k \neq 2$ and take $X$ to be the 4 points $(0:-1: 1),(0: 0: 1),(0: 1: 1),(1: 0: 1)$ in $\mathbb{P}_{k}^{2}$. These points and their minimal separators are depicted in the following figure:

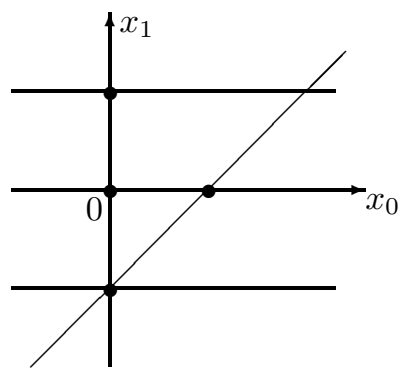


Notice that

$$
R=k\left[x_{0}, x_{1}, x_{2}\right] /\left(x_{0} x_{1}, x_{0}\left(x_{0}-x_{2}\right), x_{1}\left(x_{1}-x_{2}\right)\left(x_{1}+x_{2}\right)\right),
$$

and one easily sees that $a=1$. We choose $y$ to be the image of $x_{2}$, and as minimal separators of $X$ we take $x_{1}\left(x_{1}-x_{2}\right),\left(x_{0}-x_{1}-x_{2}\right)\left(x_{1}-x_{2}\right), x_{1}\left(x_{1}+x_{2}\right), x_{0}$. From the geometric interpretation of the core in terms of separators, Proposition 5.3(a), one immediately sees that $\operatorname{core}(X)=\mathfrak{m}^{3}+x_{0}^{2} R \supsetneq \mathfrak{m}^{3}=\mathfrak{m}^{a+2}$. The inclusion $x_{0}^{2} R \subset \operatorname{core}(X)$ would have also been predicted by Corollary 5.4 with $f=x_{0}$, and the strict containment $\mathfrak{m}^{3} \subsetneq \operatorname{core}(X)$ reflects the obvious fact that $X$ does not have the Cayley-Bacharach property.

Corollary 5.7. Let $k$ be a field of characteristic zero, let $Y \subset \mathbb{P}_{k}^{n+1}$ be a reduced and irreducible arithmetically Cohen-Macaulay curve, and write $\mathfrak{n}$ for the homogeneous maximal ideal of the homogeneous coordinate ring of $Y$. Consider a general hyperplane section $X \subset \mathbb{P}_{k}^{n}$ of $Y$ and use the notation of 5.1. One has $\operatorname{core}(Y)=\operatorname{core}(\mathfrak{n})=\mathfrak{n}^{a+2}$ and $\operatorname{core}(X)=\mathfrak{m}^{a+2}$.

Proof. According to [9, 3.4] the set of points $X$ has the Cayley-Bacharach property. Now the two equalities follow from Corollaries 4.3 and 5.5 .

\section{LOCAL ESTIMATES ON CORES}

We finish this paper with a generalization of Corollary 5.4 to the context of zero-dimensional ideals in local rings.

Proposition 6.1. Let $(R, \mathfrak{m})$ be a local Cohen-Macaulay ring with infinite residue field, $I$ an $\mathfrak{m}$-primary $R$-ideal, and $L$ and $H$ two $R$-ideals such that $L H=0$ in $R$. Write $e=e(I ; R / H)$ for the multiplicity of the ring $R / H$ with respect to the ideal I. Then $L I^{e} \subset \operatorname{core}(I)$.

Proof. We prove the claim by induction on $d=\operatorname{dim} R$. If $d=0$, then $e$ is the length of $R / H$, and we easily obtain $I^{e} \subset H$. Therefore $L I^{e} \subset L H=0$. Now consider the case $d \geq 1$. For $J$ an arbitrary reduction of $I$ we need to show that $L I^{e} \subset J$. Let $x$ be a general element of $J$ and write ${ }^{-}$for images in the $(d-1)$ dimensional Cohen-Macaulay ring $\bar{R}=R /(x)$. Notice that $L H^{\mathrm{unm}}=0$ since $R$ is Cohen-Macaulay and that $e\left(I ; R / H^{\mathrm{unm}}\right)=e$ by the associativity formula for the Hilbert-Samuel multiplicity. Thus we may replace $H$ by $H^{\text {unm }}$ to assume that $H$ is unmixed. We may further suppose that $L \neq 0$. Therefore $\operatorname{dim} R / H=\operatorname{dim} R=d$ since $R$ is Cohen-Macaulay. We conclude that the general element $x$ of $J$ is regular on $R / H$. Thus $e(\bar{J} ; \bar{R} / \bar{H})=e(J ; R / H)$. As $\bar{J}$ and $J$ are reductions of $\bar{I}$ and $I$, respectively, we have $e(\bar{J} ; \bar{R} / \bar{H})=e(\bar{I} ; \bar{R} / \bar{H})$ and $e(J ; R / H)=e(I ; R / H)$. It follows that $e(\bar{I} ; \bar{R} / \bar{H})=e(I ; R / H)=e$. Now our induction hypothesis gives $\overline{L I^{e}} \subset \operatorname{core}(\bar{I}) \subset \bar{J}$. Hence indeed $L I^{e} \subset J$.

We obtain the estimate $f \mathfrak{m}^{e} \subset \operatorname{core}(X)$ of Corollary 5.4 from the above proposition if we take $L=f R$ and $H=I_{Z} R$.

\section{REFERENCES}

[1] W. Bruns and J. Herzog, Cohen-Macaulay Rings, revised edition, Cambridge University Press, Cambridge, 1998. MR1251956 (95h:13020)

[2] M. Chardin and B. Ulrich, Liaison and Castelnuovo-Mumford regularity, Amer. J. Math. 124 (2002), 1103-1124. MR1939782 (2004c:14095) 
[3] A. Corso, C. Polini, and B. Ulrich, The structure of the core of ideals, Math. Ann. 321 (2001), 89-105. MR 1857370 (2002j:13005)

[4] C. Cumming, The canonical module of the extended Rees ring, preprint.

[5] F. El Zein, Complexe dualisant et applications à la classe fondamentale d'un cycle, Bull. Soc. Math. France 58 (1978). MR0518299 (80h:14009)

[6] E. G. Evans and P. Griffith, The syzygy problem, Ann. of Math. (2) 114 (1981), 323-333. MR632842 (83i:13006)

[7] L. Fouli, C. Polini, and B. Ulrich, The core of ideals in arbitrary characteristic, Michigan Math. J. 57 (2008), 305-319.

[8] A. Geramita, M. Kreuzer, and L. Robbiano, Cayley-Bacharach schemes and their canonical modules, Trans. Amer. Math. Soc. 339 (1993), 163-189. MR.1102886 (93k:14065)

[9] J. Harris, Curves in projective space, With the collaboration of David Eisenbud, Séminaire de Mathématiques Supérieures 85, Presses de l'Université de Montréal (1982). MR685427 (84g:14024)

[10] E. Hyry and K. E. Smith, On a non-vanishing conjecture of Kawamata and the core of an ideal, Amer. J. Math. 125 (2003), 1349-1410. MR2018664(2006c:13036)

[11] E. Hyry and K. E. Smith, Core versus graded core, and global sections of line bundles, Trans. Amer. Math. Soc. 356 (2004), 3143-3166. MR2052944 (2005g:13007)

[12] E. Kunz and R. Waldi, Regular differential forms, Contemp. Math. 79 (1988). MR971502 (90a:14021)

[13] J. Lipman, Dualizing sheaves, differentials and residues on algebraic varieties, Astérisque 117 (1984). MR759943 (86g:14008)

[14] C. Polini and B. Ulrich, A formula for the core of an ideal, Math. Ann. 331 (2005), 487-503. MR2122537 (2006k:13020)

[15] C. Polini, B. Ulrich, and M. A. Vitulli, The core of zero-dimensional monomial ideals, Adv. Math. 211 (2007), 72-93. MR.2313528 (2008b:13033)

[16] B. Ulrich, Artin-Nagata properties and reductions of ideals, Contemp. Math. 159 (1994), 373-400. MR1266194 (95a:13017)

Department of Mathematical Sciences, New Mexico State University, Las Cruces, New MeXico 88003

E-mail address: lfouli@math.nmsu.edu

Department of Mathematics, University of Notre Dame, Notre Dame, Indiana 46556

E-mail address: cpolini@nd.edu

Department of Mathematics, Purdue University, West Lafayette, Indiana 47907

E-mail address: ulrich@math.purdue.edu 